\title{
A GENERALIZATION OF SMITH THEORY
}

\author{
J. P. MAY
}

(Communicated by Haynes R. Miller)

\begin{abstract}
Using Bredon cohomology, new relations are obtained between the $\bmod p$ Betti numbers of a finite $G-C W$ complex and its singular subspace, where $G$ is a finite $p$-group.
\end{abstract}

Let $G$ be a $p$-group of finite order $p^{e}$ and let $X$ be a finite dimensional $G$-CW complex such that $H^{*}(X)$ is finite dimensional, where cohomology is understood with $\bmod p$ coefficients. Let $S X$ denote the subcomplex of singular points of $X$, that is, of points fixed by some $g \neq e$. Finally, let $F X=X / S X ; F X$ is a based $G$-CW complex such that the action off the basepoint is free. We seek relations among the $\bmod p$ Betti numbers

$$
a_{q}=\operatorname{dim} \tilde{H}^{q}(F X / G), \quad b_{q}=\operatorname{dim} H^{q}(X), \quad \text { and } \quad c_{q}=\operatorname{dim} H^{q}(S X) .
$$

If $G$ is cyclic of order $p$, so that $S X=X^{G}$, Floyd's formulation [2, 4.4] of Smith theory gives the following inequality for $q \geq 0$ and $r \geq 0$, where $r$ is odd if $p$ is odd:

$$
a_{q}+c_{q}+c_{q+1}+\cdots+c_{q+r} \leq b_{q}+b_{q+1}+\cdots+b_{q+r}+a_{q+r+1} .
$$

Floyd [2, p. 146] also gives the Euler characteristic relation

$$
\chi(X)=\chi\left(X^{G}\right)+p \tilde{\chi}(F X / G),
$$

where the reduced Euler characteristic of a based space is one less than the actual Euler characteristic. With $q=0$ and $r$ large, $(*)$ gives $\sum c_{q} \leq \sum b_{q}$. When $X$ is a $\bmod p$ cohomology sphere, the last inequality and the relation $\chi(X) \equiv \chi\left(X^{G}\right)$ $\bmod p$ immediately imply Smith's conclusion that $X^{G}$ is also a $\bmod p$ cohomology sphere.

In the general case, classical Smith theory and induction on $e$ imply dimensional restrictions on the cohomology of all fixed point spaces $X^{H}$ and therefore, by inductive use of Mayer-Vietoris sequences, on the cohomology of $S X$. Our new observation is that much sharper dimensional restrictions can be derived directly.

THEOREM. The following inequality holds for any $q \geq 0$ and $r \geq 0$ :

$$
a_{q}+\sum_{i=0}^{r}\left(p^{e}-1\right)^{i} c_{q+i} \leq \sum_{i=0}^{r}\left(p^{e}-1\right)^{i} b_{q+i}+\left(p^{e}-1\right)^{r+1} a_{q+r+1} .
$$

In particular, with $r$ large,

$$
\sum_{i \geq 0}\left(p^{e}-1\right)^{i} c_{q+i} \leq \sum_{i \geq 0}\left(p^{e}-1\right)^{i} b_{q+i}
$$

Received by the editors August 15, 1986.

1980 Mathematics Subject Classification (1985 Revision). Primary 57S17; Secondary 55N25.

Key words and phrases. Smith theory, $G$-CW complexes, finite p-groups, Bredon cohomology. 
Moreover,

$$
\chi(X)=\chi(S X)+p^{e} \tilde{\chi}(F X / G) .
$$

Since $a_{q}=0$ for $q$ large by the finite dimensionality of $X$, the inequalities and the finiteness of the $b_{q}$ imply the finiteness of the $a_{q}$ and $c_{q}$. Of course, the Euler characteristic formula is trivial when $X$ is a finite $G$-CW complex. If $p=2$, the inequalities in the classical case $e=1$ are those given by Floyd. If $p>2$, the inequalities in the case $e=1$ differ from those of Floyd due to the coefficients $(p-1)^{i}$. We shall explain after the proof why the cyclic groups of odd prime order behave exceptionally.

When $G$ is cyclic, our inequalities do not appear to give new information; in the noncyclic case, they do. For example, our inequalities obviously imply that if $c_{q}=b_{q}$ for all $q>n$, then $c_{n} \leq b_{n}$. Even this simple fact does not seem to follow from any previous version of Smith theory.

To prove the theorem, observe first that the inequalities for $r>0$ will follow inductively from those for $r=0$, which read

$$
a_{q}+c_{q} \leq b_{q}+\left(p^{e}-1\right) a_{q+1} .
$$

To obtain the inequalities for $r=1$, we add $\left(p^{e}-1\right) c_{q+1}$ to both sides, and so on.

The proof of the theorem is an application of Bredon cohomology [1]. Let $G O$ denote the category of orbits $G / H$ and $G$-maps between them. A coefficient system is a contravariant functor from $G O$ to the category of Abelian groups. For each coefficient system $M$, there is a cohomology theory $H_{G}^{*}(? ; M)$ on $G$-CW complexes. It is characterized by a dimension axiom: when restricted to the category $G O$, $H_{G}^{q}(? ; M)$ is the coefficient system $M$ if $q=0$ and is identically zero if $q \neq 0$. An exact sequence of coefficient systems $0 \rightarrow L \rightarrow M \rightarrow N \rightarrow 0$ gives rise to a natural long exact sequence

$$
\cdots \rightarrow H_{G}^{q}(X ; L) \rightarrow H_{G}^{q}(X ; M) \rightarrow H_{G}^{q}(X ; N) \rightarrow H_{G}^{q+1}(X ; L) \rightarrow \cdots .
$$

There are coefficient systems $L, M$, and $N$ such that

$$
H_{G}^{q}(X ; L) \cong \tilde{H}^{q}(F X / G), \quad H_{G}^{q}(X ; M) \cong H^{q}(X), \quad \text { and } \quad H_{G}^{q}(X ; N) \cong H^{q}(S X) .
$$

In fact, to specify $L, M$, and $N$, we can and must set

$$
L(?)=\tilde{H}^{0}(F ? / G), \quad M(?)=H^{0}(?), \quad \text { and } \quad N(?)=H^{0}(S ?)
$$

on orbits and on $G$-maps between orbits. Thus $L(G)=Z_{p}$ and $L(G / H)=0$ if $H \neq e ; M(G / H)=Z_{p}[G / H]$ for all $H$; and $N(G)=0$ and $N(G / H)=Z_{p}[G / H]$ if $H \neq e$. In particular, $M(G)$ is the group ring $Z_{p}[G]$ regarded as a $G$-module.

Let $I$ be the augmentation ideal in $Z_{p}[G]$, let $s$ be maximal such that $I^{s} \neq 0$, and let $d_{n}$ be the dimension of the $Z_{p}$-vector space $I^{n} / I^{n+1}$ for $1 \leq n \leq s$. The values of the $d_{n}$ are given by Jennings' formula $[3,2.10]$, but we only need the relations $d_{s}=1$ and $\sum d_{n}=p^{e}-1$. Write $I^{n}$ ambiguously for both the ideal and the coefficient system with $I^{n}(G)=I^{n}$ and $I^{n}(G / H)=0$ for $H \neq e$. Then $I$ is a subcoefficient system of $M$, and $M / I=L \oplus N$ since $Z_{p}[G] / I \cong Z_{p}$ and since the map

$$
Z_{p}[G / H]=H^{0}(G / H) \rightarrow H^{0}(G)=Z_{p}[G]
$$


induced by a $G$-map $G \rightarrow G / H$ with $H \neq G$ takes values in $I$. The long exact cohomology sequence associated to the short exact sequence

$$
0 \rightarrow I \rightarrow M \rightarrow L \oplus N \rightarrow 0
$$

gives the inequality

$$
a_{q}+c_{q} \leq b_{q}+\operatorname{dim} H_{G}^{q+1}(X ; I)
$$

and the Euler characteristic formula

$$
\chi(X)=\chi(S X)+\chi(F X / G)+\chi\left(H_{G}^{*}(X ; I)\right) .
$$

For $1 \leq n<s$, we have an evident short exact sequence

$$
0 \rightarrow I^{n+1} \rightarrow I^{n} \rightarrow d_{n} L \rightarrow 0,
$$

where $d L$ denotes the direct sum of $d$ copies of $L$. The resulting long exact sequence in cohomology gives

$$
\operatorname{dim} H_{G}^{q}\left(X ; I^{n}\right) \leq d_{n} a_{q}+\operatorname{dim} H_{G}^{q}\left(X ; I^{n+1}\right)
$$

and

$$
\chi\left(H_{G}^{*}\left(X ; I^{n}\right)\right)=d_{n} \tilde{\chi}(F X / G)+\chi\left(H_{G}^{*}\left(X ; I^{n+1}\right)\right) .
$$

Since $d_{s}=1, I^{s}=L$ and $H_{G}^{q}\left(X ; I^{s}\right)=\tilde{H}^{q}(F X / G)$. Our theorem follows.

If $G$ is cyclic of odd prime order $p$, then $s=p-1$ and $I^{p-1}=L$. Here $Z_{p}[G] / I^{p-1} \cong I$ as $Z_{p}[G]$-modules. If $t$ generates $G$, then the norm $\sum t^{i}$ generates $I^{p-1}$, and it follows that $M / I^{p-1} \cong I \oplus N$ as coefficient systems. We thus have short exact sequences

$$
0 \rightarrow I \rightarrow M \rightarrow L \oplus N \rightarrow 0 \text { and } 0 \rightarrow L \rightarrow M \rightarrow I \oplus N \rightarrow 0 .
$$

With $\bar{a}_{q}=\operatorname{dim} H_{G}^{q}(X ; I)$, these imply the two inequalities

$$
a_{q}+c_{q} \leq b_{q}+\bar{a}_{q+1} \text { and } \bar{a}_{q}+c_{q} \leq b_{q}+a_{q+1} .
$$

Floyd's inequalities $(*)$ follow inductively.

It is a pleasure to thank Jim McClure for teaching me the Bredon cohomology approach to classical Smith theory.

\section{BIBLIOGRAPHY}

1. G. E. Bredon, Equivariant cohomology theories, Lecture Notes in Math., vol. 34, SpringerVerlag, Berlin and New York, 1967.

2. E. E. Floyd, On periodic maps and the Euler characteristics of associated spaces, Trans. Amer. Math. Soc. 72 (1952), 138-147.

3. B. Huppert and N. Blackburn, Finite groups. II, Springer-Verlag, Berlin and New York, 1982.

Department of Mathematics, University of Chicago, Chicago, Illinois 60637 